\title{
La educación social en Europa y Costa Rica. Las funciones latentes de Merton en los servicios públicos de intervención social
}

\section{Social education in Europe and Costa Rica. Merton's latent functions in public social intervention services}

\author{
Ana Isabel Fernández Laso \\ anaislaso@gmail.com \\ Doctorado en Ciencias Sociales, Universidad Nacional, Costa Rica
}

Recibido: 15/10/2019 • Aceptado: 16/11/2020

\section{RESUMEN}

El paradigma funcionalista, a pesar de las críticas recibidas, continúa vigente en el panorama actual de las Ciencias Sociales. Robert K. Merton, entre las contribuciones que realiza al funcionalismo, conceptualiza como funciones manifiestas a las consecuencias objetivas observadas que son buscadas y reconocidas; también, denomina funciones latentes a aquellas que no lo son. Esta distinción puede facilitar una vía metodológica para el análisis funcional de las instituciones sociales y conducir hacia la observación y posterior estudio de sus funciones latentes. La Educación Social es una profesión y una metodología de la intervención en el contexto de lo social. Partiendo de sus funciones manifiestas, se realiza una propuesta de análisis de las posibles funciones latentes de las instituciones públicas donde se desarrolla. 
Palabras clave: Educación Social, funcionalismo, instituciones sociales, funciones manifiestas, funciones latentes, intervención social, observación.

\section{ABSTRACT}

The functionalist or utilitarian paradigm, despite the critics received, is still in force in the current situation of social sciences. Robert K. Merton, among his contributions to functionalism, conceptualizes as manifest functions the observed and objective consequences which are searched for and recognized. He names latent functions those which are not. This distinction can provide with a methodological way for the functional analysis of our social institutions and lead us towards the observation and later study of their latent functions. Social Education is a profession and intervention methodology in social context. Starting from its manifest functions, a proposal is made to analyze the possible latent functions of the public institutions where it is carried out.

Keywords: Social Education, functionalism, social institutions, manifest functions, latent functions, social intervention, observation.

\section{INTRODUCCIÓN}

"Lo que acostumbramos a llamar instituciones necesarias, muchas veces son instituciones a las que nos hemos acostumbrado"

Alexis de Tocqueville (2018)

La Educación Social es una carrera universitaria y una profesión reconocida en Europa desde hace décadas. En sus correspondientes denominaciones, al menos en la mitad de los países europeos se imparte esta formación especializada dentro del nivel académico de estudios superiores. En España, 39 universidades imparten actualmente esta titulación. Su ámbito de intervención se encuentra ubicado en el trabajo de lo social y, por tanto, interviene prioritariamente en colectivos de especial vulnerabilidad (adolescencia, diversidad funcional, tercera edad, minorías étnicas, centros de acogida, víctimas de violencia, etc.). 
En los países centroamericanos, no parece existir una cualificación profesional homóloga, donde además, cuando aparece el término educación social hace referencia a una profesión docente, claramente diferenciada, que se ubica en el entorno educativo formal. En el territorio de América Central, la intervención socioeducativa que realizan profesionales de la Educación Social en Europa, estaría muy próxima a las planificadas, ejecutadas y evaluadas por quienes son especialistas en Trabajo Social y Psicología Social.

Teniendo en cuenta que en este artículo se hace referencia, por tanto, a la Educación Social como proceso de intervención, se ha intentado proceder a una reflexión de cómo las teorías de Robert K. Merton podrían aportar un análisis profundo a las instituciones públicas en cuanto a las funciones latentes desarrolladas en el entorno social. Para ello, se plantea el artículo en dos bloques principales. El primero realiza una aproximación a la profesión y función de la Educación Social, con el objeto de dar a conocer esta formación académica y problematizar sobre ella. En el segundo bloque se presenta una propuesta de observación para el análisis de funciones latentes en las instituciones de intervención social del sector público, partiendo de las premisas de Robert King Merton (1910-2003).

Este sociólogo estadounidense aparece referenciado en más de 13 millones de sitios web de todo el mundo. Solo durante el último año, se pueden encontrar cerca de 4 mil textos digitalizados que citan su obra, lo que evidencia el interés que suscitan en la actualidad las teorías desarrolladas por este autor.

\section{LA EDUCACIÓN SOCIAL}

Es inevitable comenzar este artículo delimitando conceptualmente la Educación Social. Aunque en Europa se encuentran numerosas definiciones en torno a la Educación Social, para centrar la lectura, se puede realizar un primer acercamiento al concepto a través de la propuesta que realiza la Plataforma Común de la AIEJI ${ }^{1}$, que la define así: "la teoría de cómo las condiciones psicológicas, sociales y materiales, y diferentes orientaciones

1 AIEJI Asociación Internacional de Educadores Sociales fundada en 1951 en Alemania, es considerada pionera en estudios y divulgación sobre Educación Social. 
de valores promueven o dificultan el desarrollo y el crecimiento, la calidad de vida y el bienestar del individuo o del grupo" (2005).

El portal web Eduso², en representación de los diferentes Colegios Profesionales españoles, define la Educación Social como:

Derecho de la ciudadanía que se concreta en el reconocimiento de una profesión de carácter pedagógico, generadora de contextos educativos y acciones mediadoras y formativas, que son ámbito de competencia profesional del educador social, posibilitando:

La incorporación del sujeto de la educación a la diversidad de las redes sociales, entendida como el desarrollo de la sociabilidad y la circulación social.

- La promoción cultural y social, entendida como apertura a nuevas posibilidades de la adquisición de bienes culturales, que amplíen las perspectivas educativas, laborales, de ocio y participación social (www.eduso.net/red/definicion).

Fermoso (2003) propone el término de Ciencia de la Educación Social (¿Pedagogía Social o Ciencia de la Educación Social?) y lo delimita socialmente en tres sentidos: " $1^{\mathrm{a}}$ ) como teoría científica sobre la educación social; $2^{\mathrm{a}}$ ) como asignatura o disciplina académica, en el Currículo, y $3^{\mathrm{a}}$ ) como praxis o actividad profesional, ejercida con intervenciones técnicas" (s.f., p. 64).

Este autor considera como válida la acepción primera, conocimiento científico sobre la educación; sin embargo, para este artículo procede subrayar la tercera acepción, que facilita la toma de contacto con este ámbito laboral encontrado en Europa, como praxis o actividad profesional. En todo caso, la Ciencia de la Educación Social es tratada por diversas disciplinas, lo que resulta un análisis interesante para profundizar en este y otros autores que abordan la temática, pero que no compete en esta ocasión.

Por tanto, a modo de resumen de las propuestas conceptuales abordadas en este apartado, cabe destacar la relevancia que adquiere la metodología

2 Se trata del portal web del Consejo General de Colegios de Educadoras y Educadores Sociales (CGCEES). La Revista RES es la publicación digital del mismo y forma parte del proyecto EDUSO y se integra en el Portal de la Educación Social. 
a la hora de definir la Educación Social. Así, una delimitación inicial es que cuando se habla de Educación Social, se hace referencia a la praxis (en niveles de planificación, ejecución y/o evaluación) de planes, programas y/o proyectos cuyo fin es el de mejorar la calidad de vida de las personas, principalmente a través de la participación social.

\section{El debate académico}

En cierta medida, la confusión respecto a la terminología social surge del debate académico o teórico, que guarda una estrecha relación con la práctica profesional. Este se sustenta en las diferentes concepciones de la humanidad, de la sociedad, de la ciencia, de las ideas y de las ideologías.

Por este motivo, llegado este punto en las delimitaciones conceptuales, es más que razonable afrontar el debate académico sobre la Pedagogía Social y la Educación Social. A priori, la más comúnmente aceptada sería considerar a la Educación Social como el fenómeno, la realidad, la praxis y la acción, y considerar la Pedagogía Social como la reflexión y la disciplina científica (Ortega, 2004).

\section{Social Work. Aproximación epistemológica y diálogo entre disciplinas}

Para finalizar este breve acercamiento a la disciplina de la Educación Social, se va a exponer de manera sucinta una de las confrontaciones clásicas en esta cuestión. No sería razonable, para cualquier texto que aborde esta temática de la Educación Social, obviar el debate que subyace desde hace décadas, entre las disciplinas de la Educación Social y del Trabajo Social.

Para desarrollar las aclaraciones pertinentes al respecto, en este artículo se parte del detallado estudio realizado por el Consejo General de Colegios de Educadoras y Educadores Sociales (CGCEES), La profesión de la Educación Social en Europa, publicado en el año 2013. En él, se identifican básicamente dos interpretaciones sobre el ámbito profesional del término Social Work. La primera de ellas se basa en la traducción textual de "Trabajo Social". En la segunda, Social Work es entendido como "Trabajo de lo Social" o "Trabajo dentro del ámbito social". Por lo tanto, la segunda interpretación de mayor amplitud, permite incorporar a la Educación Social. El análisis comparado realizado por el CGCEES, tal y como sus 
autoras indican, "parte desde un enfoque interpretativo" (Calderón, 2013, p. 15) que pretende dilucidar los matices ocultos cuando se producen las traducciones al inglés. Estas diferencias se pueden encontrar, en relación con el Social Work, de la siguiente manera:

Social Work se define simultáneamente como ciencia, teoría y actividad profesional. Está aceptado que la Pedagogía Social y el Social Work son ciencias. Las posiciones en Europa son diversas, ya que hay algunos que aceptan que el concepto de Social Work implica el carácter educativo. (Marynowicz-Hetka citada en Calderón, 2013, p. 19).

Para resolver el problema epistemológico que plantea la Educación Social frente al concepto de Social Work en Europa, numerosos autores (Quintana, 1988; Hämälaien, 2003) han realizado una síntesis de la historia de la dilucidación de este problema, que el estudio comparado del CGCEES (2013) resume así:

El "teorema de la divergencia": determina que se trata de nociones claramente diferenciadas, con ámbitos profesionales propios.

El "teorema de la convergencia": ambos términos y sus consiguientes funciones laborales son equivalentes.

El "teorema de la subordinación": el trabajo social es un concepto más amplio que el de educación social y, por lo tanto, el primero incluye al segundo.

Existe un amplio abanico de autores/as que se posicionan firmemente desde alguno de estos teoremas. Worebcke (2003), por ejemplo, defiende el primero de ellos, mientras que Labonté (2006) considera que existe un campo profesional definido para cada disciplina con claras perspectivas de futuro en cada una de ellas.

El debate abierto entre disciplinas se ha expuesto aquí con el objeto de recalcar que ni siquiera hay consenso que pueda aplicarse al panorama europeo profesional en su totalidad, a pesar del tiempo que lleva abordándose la cuestión. Por consiguiente, esta visión de pluralidad ofrece un anticipo acerca de la dificultad para encontrar acuerdos epistemológicos en otros territorios. 
Si se habla del ámbito profesional de la Educación Social en Costa Rica, claramente, hay una ocupación que actualmente no está definida como tal y que ejercen profesionales en trabajado sociales y docencia comunitaria principalmente, como se adelantaba en la introducción del artículo. Las Enseñanzas en Estudios Sociales y Educación Cívica que imparten las universidades costarricenses nada tienen que ver con la Educación Social europea. La diferencia aquí no está solo en la terminología con la que se refiere la figura profesional sino en las funciones que desempeñan y a la preparación académica en sus universidades, pues quienes se gradúan en Educación Social en Europa han estudiado asignaturas del ámbito del trabajo social, de la psicología y de la pedagogía, ya que como se mencionaba anteriormente, la Educación Social es una metodología de la intervención social y estas asignaturas tienen una carga importante en los programas académicos dirigidos a estas/os profesionales. Por otro lado, la práctica de la Educación Social no está vinculada al sistema educativo formal, aunque es cierto que algunos territorios europeos han incorporado estos perfiles en sus centros educativos para desempeñar funciones similares a las que el Profesor Comunitario está desempeñando en Costa Rica.

Una vez delimitada la cuestión epistemológica de la Educación Social, cabe plantearse cuáles son las funciones que deben desempeñar sus profesionales en las instituciones públicas para las que trabajan. Si bien parece obvio que el propósito de esta organización es el de disminuir, si no erradicar, las situaciones de desventaja social, por todo el planeta se encuentran infinidad de programas sociales que, en lugar de disminuir la población con la que "intervienen" año tras año, según se desarrollan estas políticas en cuestión, parece que cada vez existe mayor número de población que requiere de estos servicios. Dicho de otra manera, parece darse una dualidad, en lo que a funciones sociales se refiere, dentro de la política social, ya que en lugar de erradicar la pobreza, parecen sostenerla y, de hecho, aumenta con el paso del tiempo.

\section{EL PARADIGMA FUNCIONALISTA. EL PAPEL DE LAS INSTITUCIONES Y LAS FUNCIONES LATENTES DE ROBERT K. MERTON}

La idea central del funcionalismo es el equilibrio social, "(...) adopta un modelo organicista de sociedad, donde el concepto de función sirve para explicar las relaciones entre un todo (organismo) y sus partes diferenciadas (órganos)" (Cadenas, 2016, p. 2). Las tensiones y relaciones de sus partes 
se definen como "necesidad". Las instituciones surgen para dar respuesta a estas demandas, tratando de satisfacer las necesidades. Su finalidad es la de mantener y reproducir el sistema. Consiguiendo dar respuesta a las demandas, el sistema se mantiene en equilibrio y progresa. Esta idea, precisamente, lleva al funcionalismo a concebir a las instituciones como respuesta positiva del sistema; el funcionalismo, por ende, no cuestiona el sistema. Las sociedades avanzan sin dañar el orden social, el equilibrio. Parsons (1970), desde el enfoque estructural funcionalista, define este orden como valores generales compartidos por los individuos.

El funcionalismo recibe críticas por su carácter simplista, por su prepotencia a la hora de explicar el mundo y por la falta de movilidad, al entender como disfuncional a cualquier elemento o proceso que no se ajusta al orden social. Conviene recordar aquí, que no es objeto de este artículo cuestionar el paradigma ni a sus detractores. El foco de atención se postula en algunas de las aportaciones realizadas al funcionalismo por el sociólogo estadounidense Robert K. Merton. El paradigma funcionalista atribuye una función social a todos los individuos que componen una comunidad. Sin embargo, Merton va a cuestionar que exista esta función social de todos los miembros y va a distinguir los tipos de funciones que las cosas pueden desempeñar. Según este autor, las funciones no siempre son las previstas por quien las ha diseñado, pero, incluso en el caso de que lo sean, pueden estar subyaciendo otras funciones de igual o mayor relevancia.

A pesar de las críticas mencionadas, parece incuestionable la repercusión del funcionalismo en las estructuras sociales vigentes. Las instituciones, particularmente las de entidad pública, que sirven para mantener y reproducir el orden hegemónico, han sido diseñadas para cumplir una serie funciones manifiestas que cualquier usuario podría numerar. Sin embargo ¿existen estudios sobre las funciones latentes de estas instituciones? Durante el presente artículo se va a presentar una breve propuesta de observación y análisis para aquellas instituciones que tienen que ver con las disciplinas vinculadas a la intervención social. La función evidente de estas organizaciones sería la de salvaguardar, mejorar la calidad de vida y reducir progresivamente, la población que las utiliza. Según la clasificación de Merton en cuanto a las funciones, estas pueden suscitar, entre otras, las siguientes interrogantes: ¿Cumplen con una función preventiva e integradora como se presupone?, ¿se benefician de estas funciones manifiestas 
entidades o colectivos no manifiestos?, ¿se ocupan las instituciones de identificar y analizar de alguna manera estas posibles funciones latentes?

En su obra Teoría y Estructuras Sociales (Merton, 1964) presenta la crítica que algunos sociólogos realizan sobe el análisis funcional como ideología conservadora. "Así, dicen esos críticos, la teoría funcional es simplemente la orientación de los científicos sociales conservadores que defenderían el presente orden de cosas, tal como es, y que atacarían la convivencia del cambio, por moderado que fuese" (p. 110).

Merton reacciona ante los reproches al funcionalismo. Según los críticos, ellos desdeñan la advertencia de Tocqueville de no confundir lo familiar con lo necesario cuando dice "las que llamamos instituciones necesarias no son con frecuencia otra cosa que instituciones a las que estamos acostumbrados" (Tocqueville 2018, citado en Merton, 1964, p. 111), y critica duramente a Myrdal (1944) cuando este habla de la función como concepto que puede tener sentido únicamente en relación con una finalidad supuesta. Merton afirma rotundamente que esto es falso.

Quizá sea por estas afirmaciones de los críticos al funcionalismo, sin entrar aquí a profundizar ni en estas ni en otras críticas al paradigma, lo que despierta en Merton la necesidad de ahondar en definir y clarificar conceptos, en una parte importante de su obra.

\section{Los vocabularios del análisis funcional y de la intervención social}

Es fácil concordar con el autor en cuanto a la necesidad de clarificar conceptos, especialmente, por la dificultad de consenso y la ligereza con la que se tiende a usar las palabras en el ámbito de la intervención social.

Desde sus mismos comienzos, el punto de vista funcional en sociología ha caído en confusión terminológica. Con excesiva frecuencia, se ha empleado una misma palabra para simbolizar conceptos diferentes, así como el mismo concepto ha sido simbolizado por diferentes palabras. La claridad del análisis y la adecuación de la comunicación son víctimas de este frívolo uso de palabras. En ocasiones, el análisis sufre por el cambio inconsciente del contenido conceptual de una palabra dada, y la comunicación con los demás se interrumpe cuando esencialmente el 
mismo contenido es oscurecido por un grupo de palabras diferentes. (Merton, 1964, p. 92).

Así, por ejemplo, es frecuente encontrar terminologías dispares para hablar de la persona o grupos de personas a quienes se dirige una institución de servicios sociales o comunitarios: "beneficiarios/as", "sujetos de intervención", "colectivo", "clientes" o el popular término "usuarios/as".

No menos frecuente es utilizar varios términos para referirse al colectivo de profesionales que intervienen a nivel social: trabajadoras/es sociales, animadoras/es socio-culturales, pedagogas/os sociales, educadoras/es sociales, etc. Si bien es cierto, en varios contextos y en algunos países estos perfiles profesionales pueden estar bien diferenciados, también lo es la común confusión que se produce entre ellos.

Merton continúa su disertación sobre el uso de la palabra y sus conceptos analizando el término función,

Entre el numeroso grupo de palabras que se usan indiferentemente y casi sinónimamente con «función», se cuentan actualmente uso, utilidad, propósito, motivo, intención, finalidad, consecuencias. Si estas palabras, y otras análogas se usan para referirse al mismo concepto estrictamente definido, no sería muy útil, naturalmente, señalar su numerosa variedad. Pero el hecho es que el uso indisciplinado de esas palabras, con su referencia conceptual ostensiblemente análoga lleva a distanciamientos cada vez más grandes del análisis funcional estricto y riguroso. Las acepciones de cada palabra que difieren más bien que coinciden con la acepción común a todas ellas, se toman como base (en forma inconsciente) de inferencias que son cada vez más dudosas al alejarse progresivamente del concepto central de función (Merton, 1964, p. 96).

Pero el concepto de función implica el punto de vista de la persona que observa, no necesariamente el punto de vista quien participa. La expresión "función social" se refiere a consecuencias objetivas observables y no a disposiciones subjetivas (propósitos, motivos, finalidades). El hecho de no distinguir entre las consecuencias sociológicas objetivas y las disposiciones subjetivas lleva, inevitablemente, a confusión en el análisis funcional (Merton, 1964, p. 97). 
Esta reflexión de Merton, permite problematizar las instituciones vigentes de protección y asistencia social, desde su preocupación por el punto de vista de quién observa y diseña sus funciones. Es lícito plantear que las instituciones sociales actuales abordan funciones que en muchas situaciones obvian el punto de vista del colectivo al que se dirigen o, en el mejor de los casos, el punto de vista de quienes participan no supone una prioridad en el diseño de programas de estas instituciones. Resulta difícil asumir que, después de la aparición y desarrollo de las disciplinas y estudios que centran su contenido en la atención de la población más vulnerable, a día de hoy no se tenga en cuenta el punto de vista de sus participantes. En el peor de los casos, ¿sería posible que no se haya querido tener en cuenta o incluso se quiera ocultar el citado punto de vista? En el caso de que así fuera, la cuestión principal debería ser el por qué.

\section{Postulados que prevalecen en el análisis funcional}

En esencia, esos postulados sostienen, primero, que las actividades sociales o las partidas culturales estandarizadas son funcionales para todo el sistema social o cultural; segundo, que todos estos renglones sociales y culturales desempeñan funciones sociológicas; y tercero, que son, en consecuencia, indispensables (Merton, 1964, p. 98).

Atendiendo a la cuestión formulada por Merton en el apartado de los conceptos de disposiciones subjetivas (motivos propósitos) "¿En qué tipos de análisis basta con tomar motivaciones observadas como datos, como dadas, y en cuáles son consideradas apropiadamente como problemáticas, como derivables de otros datos?" (Merton, 1964, p. 125). Conviene detenerse brevemente en la exposición del autor en relación con estos conceptos, puesto que van a dar lugar a la clarificación de los términos en torno a los que gira el ensayo.

En cuanto a los conceptos de consecuencias objetivas (funciones, disfunciones) el autor afirma,

Hemos observado dos tipos predominantes de confusión que envuelven las diversas concepciones corrientes de «función». 1. La tendencia a limitar las observaciones sociológicas a las aportaciones positivas de una entidad sociológica al sistema social o cultural en que está comprendida; y 2 . La tendencia a confundir 
la categoría subjetiva de motivo, o móvil, con la categoría objetiva de función (Merton, 1964, pp. 125-126).

Es entonces cuando explicita la necesidad de proceder a realizar las distinciones conceptuales que sean adecuadas para lograr erradicar estos errores.

El primer problema exige un concepto de consecuencias múltiples y un saldo líquido de una suma o agregación de consecuencias. Funciones son las consecuencias observadas que favorecen la adaptación o ajuste de un sistema dado; y disfunciones, las consecuencias observadas que aminoran la adaptación o ajuste del sistema. Hay también la posibilidad empírica de consecuencias afuncionales, que son simplemente ajenas al sistema en estudio.

En todo caso dado, una cosa puede tener consecuencias funcionales y disfuncionales, originando el difícil e importante problema de formular cánones para valorar el saldo liquido del agregado de consecuencias. (Esto es, naturalmente, más importante en el uso del análisis funcional para orientar la formación y ejecución de una política.) El segundo problema (que nace de la fácil confusión de motivos y funciones) nos obliga a introducir una distinción conceptual entre los casos en que el propósito subjetivo coincide con la consecuencia objetiva y los casos en que divergen (Merton, 1964, p. 126).

El autor trata de aclarar conceptualmente el término función por la problemática que surge de la confusión de motivos y funciones, ya que no siempre se produce el resultado esperado como propósito subjetivo. Identifica que las instituciones no siempre cumplen con las funciones previstas y que, aunque así fuera, pueden estar cumpliendo con otras inesperadas, que además pueden resultar no observadas.

Así, distingue:

Funciones manifiestas son las consecuencias objetivas que contribuyen al ajuste o adaptación del sistema y que son buscadas y reconocidas por los participantes en el sistema; funciones latentes son, correlativamente, las no buscadas ni reconocidas. Las relaciones entre las «consecuencias imprevistas» de la acción y las «funciones latentes» pueden definirse claramente, ya que están 
implícitas en la sección precedente del paradigma. Las consecuencias no buscadas de la acción son de tres tipos:

1. las que son funcionales para un sistema deliberado, y comprenden las funciones latentes;

2. las que son disfuncionales para un sistema deliberado, y comprenden las disfunciones latentes; y

3. las que son ajenas al sistema, al cual no afectan ni funcional ni disfuncionalmente, es decir, la clase de consecuencias afuncionales que desde el punto de vista pragmático carecen de importancia (Merton, 1964, pp. 126-127).

Concluye este apartado con la siguiente interrogante en lo que a lo largo del texto el autor denomina, pregunta fundamental: "¿Cuáles son los efectos de la transformación de una función anteriormente latente en una función manifiesta (que implica el problema del papel del conocimiento en la conducta humana y los problemas de la «manipulación» de la conducta humana)?" (Merton, 1964, 127).

\section{FUNCIONES MANIFIESTAS DE LA EDUCACIÓN SOCIAL}

Cuando Merton trata su paradigma de análisis funcional, más concretamente de las cosas a las que se les atribuyen funciones, deja claro que "Todo el campo de datos sociológicos puede someterse (...) al análisis funcional. El requisito fundamental es que el objeto de análisis represente una cosa estandarizada (es decir, normada y reiterativa), (...)" (Merton, 1964, p. 125). Dentro de ese campo de lo observable ubica a los "papeles sociales, normas institucionales, procesos sociales, normas culturales, emociones culturalmente normadas, normas sociales, instrumentos de control social, etcétera" (p. 125). Sin entrar a profundizar en esta clasificación de lo observable para no extender el texto, se procede a incluir aquí las instituciones gubernamentales de intervención social y socioeducativas de carácter público.

Según la definición anteriormente expuesta por Merton, y tras realizar un breve análisis de funciones manifiestas de la intervención social, se podría generalizar las siguientes funciones comunes a la mayoría de instituciones de esta índole: 
Función preventiva: actuación precoz sobre las causas que generan problemáticas individuales y colectivas, derivadas de las relaciones humanas y del entorno social. Esta función se traduce, para los profesionales de cierta cualificación profesional, en elaborar y ejecutar proyectos de intervención para grupos de población en situaciones de riesgo social y de carencia de aplicación de los derechos humanos.

Función de intervención directa: se refiere a la atención de individuos o grupos que presentan o están en riesgo de presentar, problemas de índole social. Su objeto será potenciar el desarrollo de las capacidades y facultades de las personas para afrontar por sí mismas futuros problemas e integrarse satisfactoriamente en la vida social.

Función de planificación: elaboración y ejecución de planes, programas y proyectos de acuerdo con unos objetivos propuestos, mediante un proceso de análisis de la realidad y del cálculo de las probables evoluciones de ella. Esta función se puede desarrollar en dos niveles:

Nivel microsocial: comprende el diseño de tratamientos, intervenciones y proyectos sociales.

Nivel macrosocial: comprende el diseño de programas y servicios sociales.

Función de promoción: diseñar e implementar las políticas sociales que favorezcan la creación y reajuste de servicios y recursos adecuados para la cobertura de necesidades sociales.

Función de mediación: estas instituciones actuarán como canal intermediario cuando sea requerido, posibilitando la unión de las partes implicadas en el conflicto, con el fin de que, con su intervención, sean las propias personas interesadas quienes logren llegar a una resolución.

Función de evaluación y supervisión: ejercer el control de las tareas realizadas por los profesionales, así como contrastar los resultados obtenidos en las distintas actuaciones, incorporando las medidas que sean necesarias durante su desarrollo y no solo al finalizar los programas. 


\section{OBSERVACIÓN DE FUNCIONES LATENTES EN INSTITUCIONES DE INTERVENCIÓN SOCIAL}

"No se puede ver que no se ve lo que no se ve"

Heinz Von Foerster(1979)

Sin pretensión alguna de llegar, con estas breves reflexiones a donde otros/as escribieron importantes disertaciones, el artículo pretende recordar lo complejo que puede resultar observar lo que no fue diseñado para serlo (incluso quizá el interés de su diseño esté precisamente en que no sea observado). Treinta años después de las contribuciones de Merton (1964) al funcionalismo que aquí se ocupa, Luhmann (1998) insistiría en que la sociología debe preguntarse cómo es posible observar las estructuras latentes.

Teniendo en cuenta las funciones manifiestas de la intervención social previamente descritas, se recoge la propuesta de Antonio López Piña (2017) para el desvelamiento de las funciones latentes de un comportamiento social, adaptando sus indicaciones al estudio y registro de funciones latentes al ámbito institucional de los servicios sociales y comunitarios.

El primer paso que marca el autor es registrar detallada y rigurosamente el modo en que se llevan a cabo las intervenciones, sus características de aplicación en sus términos reales y materiales. López (2017) sugiere que hay que evitar dejarse llevar "por el idealismo o acabará emergiendo a la superficie manifiesta" (parr. 11). Lo anterior es absolutamente válido para cualquier tipo de observación, ya que aunque de todos es conocido que el subjetivismo de quien observa condicionará el resultado de dicha observación, no desmerece recordarlo, especialmente en este contexto, en el que precisamente se trata de huir de las influencias de lo evidente.

López (2017) propone tres apartados dentro de este primer paso. Con la consciencia de que al trasladar al ámbito institucional el estudio queda en exceso simplificado para la envergadura que supondría tal observación, se ha adaptado su propuesta de la siguiente forma:

1. Identifique el lugar físico y orgánico (entidad, programa, proyecto, departamento, responsable, equipo) desde el que surge la intervención. 
2. Identifique las actuaciones o propuestas concretas de intervención: a quién van dirigidas (número, colectivo, características de la población diana), objetivo manifiesto, cronograma, sistema de evaluación.

3. Identifique si la actuación es novedosa o no. Cuándo se inició, quién la diseño y cómo se llevó a cabo. Periodicidad de la intervención. Evaluación de la intervención previa en el caso de que las hubiera.

El segundo paso que propone López (2017) es quizás el más relevante y el de mayor complejidad por las múltiples dimensiones del análisis, el estudio de las consecuencias económicas, sociales, culturales y políticas que se derivan de la práctica de estas intervenciones:

1. Identifique qué población, colectivos o instituciones obtienen beneficios objetivos y palpables.

2. Identifique qué población, colectivos o instituciones se ven perjudicados en el medio y largo plazo.

En el tercer y cuarto punto que propone el autor, existen dos supuestos que merece la pena analizar con mayor detenimiento:

3. Certifique cómo los grupos beneficiarios niegan cualquier ventaja asociada a la puesta en práctica de ese comportamiento.

En esta frase, por un lado, afirma con rotundidad que siempre hay un colectivo que se beneficia de la conducta (en este caso, del proyecto, servicio o intervención). Eso está muy bien, teniendo en cuenta que se están analizando instituciones de servicio público a las comunidades en vulnerabilidad social o en riesgo de estarlo. La premisa debería ser válida: siempre debería haber un colectivo o comunidad beneficiario. No obstante, no conviene dar tal hecho por sentado. La segunda presunción que hace el autor en este apartado afirma que siempre que un colectivo se beneficia, niega ante el resto de la comunidad su ventaja (López, 2017). Además, pide que se identifiquen a los miembros de esta conducta (el análisis de un comportamiento lleva a la identificación de otro). En este caso, se podría, por un lado, valorar la opción de que esto ocurra; $y$, por otro, tratar de determinar cuál es la causa que lleva al colectivo a ocultar esta ventaja. En lugar de identificar simplemente a los individuos con esta conducta, si de analizar 
un comportamiento social se trata, tendrá más consistencia para el estudio identificar sus causas, que proporciona un listado de personas.

En cualquier caso, para este punto se podría proponer otra estructura de análisis. Como se trata de funciones latentes, no será necesario identificar los colectivos que siendo usuarios del servicio se hayan visto beneficiados por él. Esto se podrá encontrar fácilmente en evaluaciones y memorias de las organizaciones. En esos documentos deberían estar incluidos, además, los casos en los que dicha función haya errado y las causas que han ocasionado, dificultado o mermado, los objetivos propuestos. Lo que habría que identificar es si existen entidades o colectivos secundarios que se benefician directa o indirectamente de la ejecución de estos proyectos y cuáles son esos beneficios (tipo de beneficio, entidades beneficiarias, si existen evaluaciones internas o externas donde se registre ese beneficio ya sea implícita o explícitamente). De igual forma, sería excelente si se da tal caso, visibilizar por qué se producen estos "beneficios colaterales".

4. Certifique cómo los grupos afectados niegan cualquier daño asociado a la puesta en práctica de ese comportamiento.

La propuesta aquí sería que, tras identificar si existen comunidades, entidades o personas que puedan verse afectadas a corto, medio y largo plazo por las intervenciones de estas instituciones, tal como se indica en el punto 2 , debe identificarse qué tipo de daño se les causa y si las instituciones tienen constancia de ellas. Igualmente, habría que consensuar qué se entiende por daño y, como se mencionaba en el apartado de las funciones de Merton, valorar si el punto de vista del colectivo usuario se ha tenido en cuenta en el planteamiento de funciones institucionales o solo se ha contado con el punto de vista de quien observa.

\section{CONCLUSIONES}

Continuando la idea del apartado anterior y especialmente del supuesto observable en el último párrafo, resulta de interés no solo para las instituciones y profesionales que trabajan en la intervención social, hacer un profundo análisis de las funciones latentes que subyacen en las instituciones. Al tratarse de organizaciones de carácter público, gestionadas por el estado y cuyo beneficiario es la ciudadanía con mayor riesgo de vulnerabilidad social, se debe considerar de interés común visibilizar si se 
producen "daños colaterales" en los proyectos, si se tiene constancia de ellos y cuáles son las repercusiones a medio y largo plazo. Recordando la cita de Tocqueville con la que comienza el artículo, podría haber multitud de instituciones que no solo no son necesarias y se mantienen por costumbre y cabría cuestionarse, incluso, si existen instituciones que, en un análisis extenso de sus funciones, puedan estar ocasionando perjuicios 0 daños a nivel comunitario o social.

Con lo anterior no se hace referencia a las llamadas políticas y medidas de discriminación positiva, cuyos fines y resultados han estado cuestionados infinidad de veces, más bien, a lo que Durkheim (1893) denominaba integración y solidaridad orgánica. Se conoce de sobra del fracaso integrador de muchas instituciones, según Durkheim, podría concluirse que, en las sociedades donde esto ocurre, el sistema falla (Merton, 2002). Sin embargo, se perpetúan políticas sociales y funciones, incluso cuando podría llegar a concluirse, tras las observaciones aquí propuestas, que algunas funciones resultasen ser más "des-integradoras" que integradoras.

No menos relevante sería el estudio y difusión de lo que anteriormente se denominó beneficios colaterales. Identificar a aquellas personas, colectivos o entidades que puedan estarse beneficiando de "forma latente" por las instituciones sociales. Lo más grave de este análisis sería descubrir que no solo se producen beneficios de este tipo en entidades secundarias (que se ven favorecidas económicamente por ciertas intervenciones y políticas) sino que, además, las instituciones fueran conscientes, aunque no lo expliciten, en sus programas y quizá podría darse el caso de que estos beneficios en realidad fueran en perjuicio de las comunidades que deberían haberse visto favorecidas originalmente. También, cabría la posibilidad de que algunas funciones latentes se hayan convertido o estén en proceso de convertirse en funciones manifiestas.

Por otra parte, cabe mencionar que una vez observadas detenidamente las funciones manifiestas de diferentes fuentes, preocupa no encontrar entre ellas ninguna que mencione la disminución progresiva de beneficiarias/ os del servicio. No se observan, ni siquiera se insinúa a modo de utopía, que la función principal de la intervención con colectivos de mayor vulnerabilidad social sea la erradicación de las propias instituciones. El éxito de sus programas debería conllevar la mejora progresiva de sus beneficiarios y, por lo tanto, la disminución del colectivo diana al que se dirigen. Esta 
situación, necesariamente llevaría a, o bien reducir el número de instituciones o servicios, o bien, la reformulación de sus funciones (por ejemplo, más centradas en la prevención y en el fomento de la participación activa de la población).

Sin embargo, la realidad de estas estructuras públicas en los servicios sociales y comunitarios es bien distinta. Cada vez tienen más demanda, aunque cada vez cuentan con menos recursos gubernamentales. Parece que la función latente fuera la de perpetuar el statu quo, la estructura social tal y como está, en lugar de erradicar, por ejemplo, la pobreza, que claramente es la función manifiesta de las políticas sociales por antonomasia. El funcionalismo atribuye una función positiva a las instituciones, al no contemplar estos asuntos, responsabilizando al individuo de su propia integración. Merton ya criticaba el "funcionalismo radical y absoluto, cuestionando los postulados de la unidad funcional de la sociedad -no todo está integrado-, del funcionalismo universal -no todo elemento de una sociedad desempeña una función-, y de la necesidad -no todo elemento es indispensable-" (Lagunas, 2016, p. 249). Plantear un análisis funcional en este sentido resultaría complejo, pero con toda seguridad, muy interesante.

Por último, poner de manifiesto la validez y vigencia del funcionalismo y sus vertientes. A pesar de las críticas a sus modelos explicativos simplistas (no se incorporan reflexiones profundas, no da cabida a las alternativas y bifurcaciones) la realidad es que este paradigma si bien puede ser funcional como explicación de conceptos sencillos, también puede ser revisado en la actualidad, como se ha podido comprobar aquí, aplicándose algunas de sus teorías y supuestos, como por ejemplo, en modelos de observación y análisis de organizaciones y sus funciones.

\section{REFERENCIAS}

Asociación Internacional de Educadores y Educadoras Sociales. (s.f.). AlEJI Latinoamérica. http://aiejilat.blogspot.com/2011/02/que-es-la-aieji.html

Cadenas, H. (2016). La función del funcionalismo: una exploración conceptual.Universidad Federal do Rio Grande do Porto Alegre.

Calderón, M. J. (Coord.). (2013). La profesión de la Educación Social en Europa. RES. https://www.eduso.net/res 
Consejo General de Colegios Oficiales de Educadoras y Educadores Sociales. (2001). Código Deontológico de la Educación Social. https:// www.eduso.net

Durkheim, E. (1893). La división del trabajo social. Madrid: Akal

Fermoso, P. (2003). ¿Pedagogía Social o Ciencia de la Educación Social? Revista interuniversitaria, (10), 61-84.

Hämälaien, J. (2003). The concept of social pedagogy in the field of social work. Journey of social work, 3(1), 69-80.

International Associaton of Social Educators. (s.f.). AIEJI. http://www.aieji.net

Labonté, C. (mayo, 2006). La formation au travail social en Europe. Acciones e investigaciones sociales. Conferencia en el VI Congreso de escuelas universitarias de trabajo social. Universidad de Zaragoza, Zaragoza.

Lagunas, D. (2016). El legado del funcionalismo. Limitaciones teóricas y excesos etnográficos. RES.

López, A. (2017). Función manifiesta y función latente. http://www.diagramadevennblog.wordpress.com

Luhmann, N. (1998). Teoría de la sociedad y pedagogía. Paidós.

Merton, R. K. (1964). Teoría y estructuras sociales. Fondo de Cultura Económica.

Merton, R. K. (2002). La división del trabajo de Durkheim. Reis.

Myrdal, G. (1944). An American dilemma. New York: Harper \& Row Publishers.

Ortega, J. (Coord.) (2004). Educación Social especializada. Ariel.

Parsons, T. (1970). The Social System. Routledge \& Kegan Paul.

Quintana, J. M. (1988). Pedagogía social. Dikynson.

Tocqueville, A. (2018). La democracia en América. Editorial Trotta. 
Von Foerster, H. (1979). Cybernetics of Cybernetics. Klaus Kripplendorff (comp.) Communication and Control in Society.

Worebcke, M. (2003). The Social Work Profession and Social Work Education in Europe. European Social Work, Annual Meeting Report, Dornbirn. 
